\title{
The first chemical synthesis of novel Me0-3-GlcuA derivative of hyaluronan-based disaccharide to elucidate the catalytic mechanism of hyaluronic acid synthases (HASs)
}

\author{
Guohua Wei ${ }^{\text {a,b }}$, Vipin Kumar ${ }^{a}$, Jun Xue ${ }^{a}$, Robert D. Locke ${ }^{a}$, Khushi L. Matta ${ }^{\text {a,* }}$ \\ a Cancer Biology, Roswell Park Cancer Institute, Buffalo, NY 14263, USA \\ ${ }^{\mathrm{b}}$ State Key laboratory of Environmental Chemistry and Ecotoxicology, Research Center for Eco-Environmental Sciences, Chinese Academy of Sciences, Beijing 100085, China
}

\section{A R T I C L E I N F O}

Article history:

Received 2 September 2009

Accepted 8 September 2009

Available online 12 September 2009

\section{Keywords:}

MeO-3-GlcUA

Hyaluronic acid synthases (HASs)

Oligosaccharides

Phosphorylation

\begin{abstract}
A B S T R A C T
The first chemical synthesis of MeO-3-GlcUA $\beta(1 \rightarrow 3)$ GlcNAc-UDP to elucidate the catalytic mechanism of hyaluronic acid synthases (HASs) is described. Construction of the desired $\beta(1 \rightarrow 3)$-linked disaccharide 10 was achieved very efficiently by coupling MeO-3-GlcUA donor $\mathbf{3}$ with the suitable protected GlcNTroc acceptor 4 using $\mathrm{BF}_{3} \cdot \mathrm{Et}_{2} \mathrm{O}$ as Lewis acid. Chemoselective removal of anomeric NAP, phosphorylation, hydrogenation, coupling with UMP-morpholidate, and finally complete deprotection gave the target compound $\mathbf{1}$ in good yield.
\end{abstract}

(c) 2009 Elsevier Ltd. All rights reserved.

\section{Introduction}

Hyaluronic acid (HA), a vital extracellular matrix component of vertebrate tissues, has been implicated in numerous physiological and biological phenomena. ${ }^{1} \mathrm{HA}$ is a linear biopolymer composed of thousands of alternating repeating disaccharide units of glucuronic acid $[\operatorname{GlcUA} \beta(1 \rightarrow 3)]$ and $N$-acetylglucosamine $[\operatorname{GlcNAc} \beta(1 \rightarrow 4)]^{2}$ The enzymes that catalyze the polymerization of HA, the hyaluronan synthases (HASs), are unique glycosyltransferases that have both selective GlcNAc $\beta(1 \rightarrow 4)$ transferase and GlcUA $\beta(1 \rightarrow 3)$ transferase activities. ${ }^{3}$ There is an ambiguity regarding the directionality of chain elongation of HA biosynthesis, that is, whether the addition of monosaccharide units occurs at the reducing or non-reducing terminus of growing polysaccharide chains. Although, many groups have investigated this problem extensively, till date there is no accurate knowledge regarding the mechanism of HA biosynthesis and hence only minimal information is available on the molecular basis for HASs catalytic action. Consequently, our understanding of the detailed mechanisms whereby hyaluronan influences cell behavior is still incomplete. ${ }^{3}$

It was identified recently that the small HA fragments behave as potent activators of immunocompetent cells which play a decisive role in the development of $\mathrm{T}$ cell-mediated immune responses. ${ }^{4}$ Also, studies such as NMR spectroscopic investigations and the mechanism of degradation, performed to understand the chemical

\footnotetext{
* Corresponding author. Fax: +1 7168458768.

E-mail address: khushi.matta@roswellpark.org (K.L. Matta).
}

properties of HA, are preferably executed using low-molecularweight HA fragments rather than polymeric HA. ${ }^{5}$ Thus, syntheses of well-defined HA fragments is required to provide adequate material for carrying out such investigations. Consequently, several strategies have been reported for the construction of HA fragments containing either a GlcUA or a GlcNAc at the reducing end. ${ }^{6}$

Moreover, it was observed that the deoxy analogs obtained by the substitution of one of the -OH groups of basic enzyme acceptor with $-\mathrm{F},-\mathrm{OMe},-\mathrm{N}_{3},-\mathrm{NH}_{2}$, etc. sometimes result in highly specific acceptors or inhibitors for the individual enzymes. ${ }^{7}$ For example, Scott and Viola found that 3-fluoro and 4-fluoro analogs of D-glucose were higher affinity substrates than D-glucose for aldolase reductase but 2-fluoro and 4-fluoro analogs of D-glucitol were inactive acceptors for sorbitol dehydrogenase. ${ }^{7 a}$ It was noticed by us that the activities of only Gal 3-O-sulfotransferases and not sialyltransferases (Sia-T) were adversely affected by $\operatorname{Gal} \beta(1 \rightarrow 4) \operatorname{GlcNAc} \beta(1 \rightarrow 6)[\mathrm{F}-3-\mathrm{Gal} \beta-$ $(1 \rightarrow 3)$ ]GalNAc $\alpha-O B n$. Amazingly, F-4-Gal $\beta(1 \rightarrow 4) \operatorname{GlcNAc} \beta(1 \rightarrow 6)-$ $[$ Gal $\beta(1 \rightarrow 3)]$ GalNAc $\alpha-O B n$ was found to be an inhibitor of $\alpha 2,6(\mathrm{~N})$ Sia-T activity but not of $\alpha 2,3(\mathrm{~N})$ Sia-T activity. ${ }^{7 \mathrm{~b}}$ The deoxy analogs of disaccharide-peracetylated GlcNAc $\beta 1-3 \mathrm{Gal} \beta-O$-naphthalenemethanol containing $-\mathrm{H},-\mathrm{F},-\mathrm{N}_{3},-\mathrm{NH}_{2}$, or $-\mathrm{OCH}_{3}$ at $\mathrm{C}-3^{\prime}$ and $\mathrm{C}-4^{\prime}$ positions of the terminal $\mathrm{N}$-acetylglucosamine residue presumably inhibit one or more galactosyltransferases in vivo, thereby blocking sLe ${ }^{\mathrm{X}}$ formation and experimental tumor cell metastasis. ${ }^{7 \mathrm{c}}$ Thus, functionalization of hydroxyl group of an HA fragment structure by substituting with $-\mathrm{F},-\mathrm{OMe},-\mathrm{N}_{3},-\mathrm{NH}_{2}$, etc. may result in highly specific acceptors or inhibitors for the individual HAS. In this context, we have reported very recently the first chemical synthesis 
of F-4-GlcUA $(1 \rightarrow 3)$ GlcNAc-UDP for investigating the catalytic mechanism of HASs. ${ }^{8}$ In general, OMe derivatives have similar potency and their synthesis requires cheap and less hazardous reagents than their respective fluoro derivatives. Therefore, we became interested in developing a facile synthesis of these OMe derivatives and report herein the first chemical synthesis of $\mathrm{MeO}-$ 3 -GlcUA $\beta(1 \rightarrow 3)$ GlcNAc-UDP $(\mathbf{1})$ which may have the potential to be a specific donor for the HASs.

\section{Results and discussion}

It was envisaged that $\mathrm{MeO}-3-\mathrm{GlcUA} \beta(1 \rightarrow 3)$ GlcNAc-1-phosphate derivative $\mathbf{2}$ would be an ideal intermediate to generate target molecule $\mathbf{1}$ (Fig. 1). The phosphate derivative $\mathbf{2}$ could in turn be obtained by the glycosylation of known GlcNTroc acceptor $\mathbf{4}^{8}$ by MeO-3-GlcUA imidate donor 3. To avoid the possible formation of side product due to transacetylation during $O$-glycosylation, benzoyl group was preferred over acetyl group for protection of the 2-OH position of GlcUA in donor 3 . $^{9}$ We preferred to utilize 2-naphthylmethyl (NAP) as an anomeric-protecting group in $\mathbf{3}$ and 4 , because of its ease in removal by DDQ oxidation. ${ }^{10}$ Due to the straightforward transformation of $\mathrm{N}$-Troc into $\mathrm{N}$-Ac, Troc group was chosen as a temporary protecting group for nitrogen in the glycosyl acceptor $4 .^{11}$

Scheme 1 outlines the synthesis of donor 3. Synthesis of imidate 3 was initiated with known GlcUA donor $\mathbf{5} .^{12}$ Induction of anomeric NAP protection in $\mathbf{5}$ was executed by the glycosylation of NAP$\mathrm{OH}$ with donor $\mathbf{5}$ in the presence of TMSOTf at $0{ }^{\circ} \mathrm{C}$ to afford $\mathbf{6}$ in $80 \%$ yield. Deacetylation of 6 with $\mathrm{NaOMe}-\mathrm{MeOH}$ followed by saponification of the methyl ester using LiOH gave $\mathbf{7}$ in good yield. 2,4-di-O-acylated derivative 8 was prepared from 7 following a 3step procedure. Lactonization of $\mathbf{7}$ with benzoic anhydride in DMF, followed by complete benzoylation using DMAP-pyridine, and finally methanolysis of the lactone ring in the presence of anhydrous $\mathrm{NaOAc}$ afforded the desired 2,4-di-O-acylated derivative 8 in 76\% yield for three steps. ${ }^{13}$ O-Methylation of the 3-hydroxyl group of 8 with $\mathrm{CH}_{3} \mathrm{I}$ and freshly prepared $\mathrm{Ag}_{2} \mathrm{O}$ provided $\mathbf{9}$ in $92 \%$ yield. Removal of anomeric NAP protection of $\mathbf{9}$ was carried out using $\mathrm{DDQ}^{10}$ followed by imidation ${ }^{14}$ to afford the MeO-3-GlcUA donor 3 in high yield (Scheme 1).

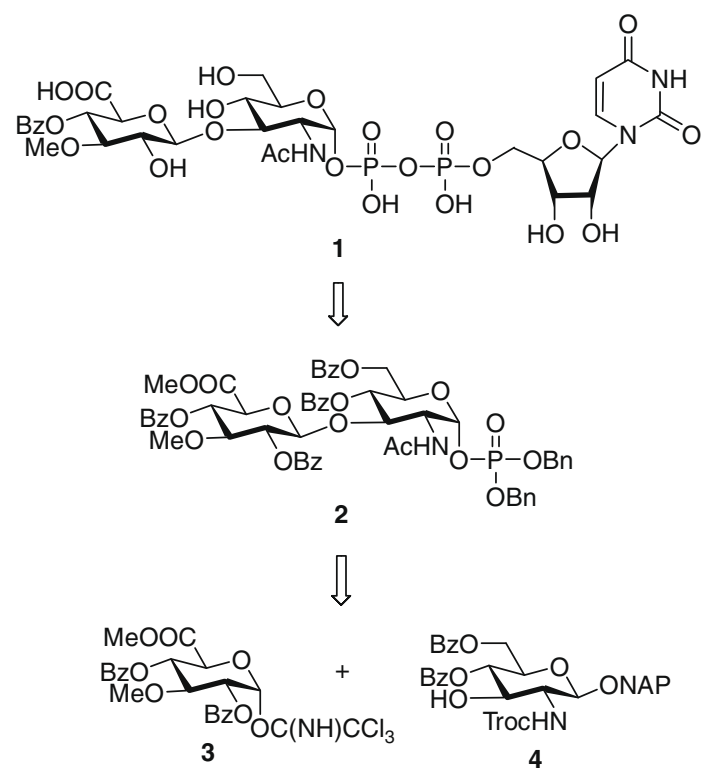

Figure 1. Retrosynthesis of target molecule MeO-3-GlcUA $\beta(1 \rightarrow 3)$ GlcNAc-UDP $(\mathbf{1})$.

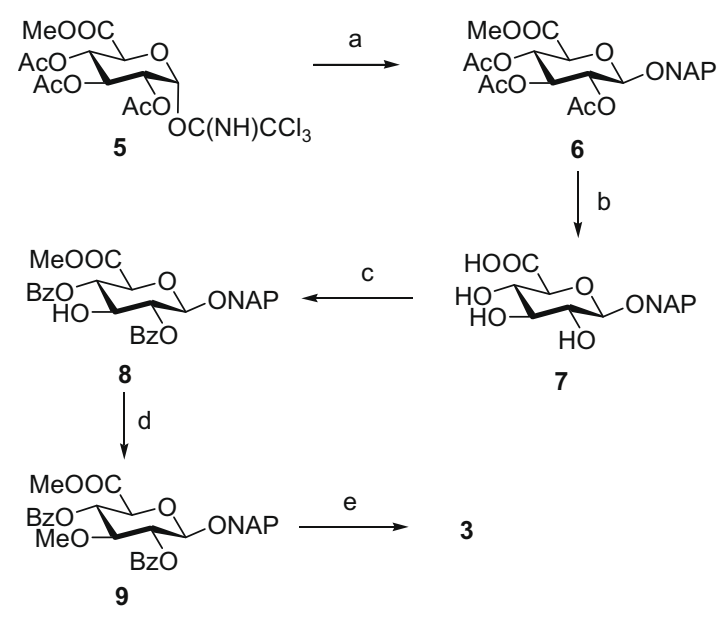

Scheme 1. Preparation of donor 3. Reagents and conditions: (a) NAPOH, TMSOTf, $\mathrm{CH}_{2} \mathrm{Cl}_{2}, 4 \AA \mathrm{MS}, 0^{\circ} \mathrm{C}, 2 \mathrm{~h}, 80 \%$; (b) (i) NaOMe, MeOH, rt, 6 h, 90\%; (ii) LiOH, THF, $\mathrm{H}_{2} \mathrm{O}$, $0{ }^{\circ} \mathrm{C}, 4 \mathrm{~h}, 95 \%$; (c) (i) $\mathrm{Bz}_{2} \mathrm{O}, \mathrm{DMF}, 85^{\circ} \mathrm{C}$, 4 h, 86\%; (ii) $\mathrm{Bz}{ }_{2} \mathrm{O}$, Py, DMAP, DMF, rt, $40 \mathrm{~h}$, 96\%; (iii) NaOAc, MeOH, DMF, rt, 10 h, 92\%; (d) MeI, $\mathrm{Ag}_{2} \mathrm{O}, \mathrm{CH}_{2} \mathrm{Cl}_{2}, 4 \AA$ molecular sieves, rt, 20 h, 92\%; (e) (i) DDQ $\mathrm{CH}_{2} \mathrm{Cl}_{2}-\mathrm{MeOH}$ (4:1), rt, 12 h, 89\%; (ii) $\mathrm{CCl}_{3} \mathrm{CN}, \mathrm{DBU}$, $\mathrm{CH}_{2} \mathrm{Cl}_{2}, 0^{\circ} \mathrm{C}, 2 \mathrm{~h}, 78 \%$

We next investigated multistep synthesis of the crucial phosphate intermediate 2 (Scheme 2). Coupling of the MeO-3-GlcUA donor 3 and the known acceptor $\mathbf{4}^{8}$ was carried out with $\mathrm{BF}_{3} \cdot \mathrm{Et}_{2} \mathrm{O}$ in the presence of $4 \AA$ molecular sieves in toluene to afford the desired disaccharide $\mathbf{1 0}$ with complete $\beta 1 \rightarrow 3$ stereoselectivity in $83 \%$ yield. ${ }^{6 e, 8,12 b, 15}$ Detrimental effect on the yield of the above-mentioned glycosylation reaction was observed upon substitution of dichloromethane as a solvent. Also, TMSOTf was found ineffective for the same glycosylation as a promoter in comparison with $\mathrm{BF}_{3} \cdot \mathrm{Et}_{2} \mathrm{O}$. It is worth mentioning that the glycosylation reaction reported here is one among the few procedures available for efficient synthesis of $\beta 1 \rightarrow 3$ linkage with high $\beta$-stereoselectivity and high yield. ${ }^{8,15 \mathrm{~d}}$ Most of the literature available on the synthesis of $\beta 1 \rightarrow 3$ linkage suffers from certain drawbacks such as poor $\beta$-selectivity and low yield. ${ }^{6 e, k, n, 12 b, 15 b, c}$ Following above glycosylation reaction, $\mathrm{MeO}-3-\mathrm{GlcUA} \beta 1 \rightarrow 3$-linked disaccharide $\mathbf{1 0}$ could be accessed with complete $\beta 1 \rightarrow 3$ stereoselectivity and in high yield. Next, replacement of the $N$-Troc group in $\mathbf{1 0}$ by an $N$-acetyl group was effected with $\mathrm{Zn}-\mathrm{Ac}_{2} \mathrm{O}$ and the subsequent acetylation of the free amine offered 11 in $88 \%$ yield. ${ }^{11}$ Chemoselective removal of NAP in 11 with DDQ in $\mathrm{CH}_{2} \mathrm{Cl}_{2}-\mathrm{MeOH}(4: 1)^{10}$ provided anomericfree hydroxyl disaccharide $\mathbf{1 2}$ in $89 \%$ yield. Phosphorylation of $\mathbf{1 2}$ with tetrabenzyl pyrophosphate ${ }^{16}$ gave the benzyl-protected anomeric phosphate $\mathbf{2}$ as the desired $\alpha$ anomer in high yield. Prominent signals in the ${ }^{1} \mathrm{H}$ NMR spectrum of $\mathbf{2}$ at $\delta=5.68$ ( $\mathrm{dd}$, $J_{1,2}=3.4 \mathrm{~Hz}, J_{1, \mathrm{P}}=6.4 \mathrm{~Hz}, 1 \mathrm{H}, \mathrm{H}-1$ of GlcNAc), and the signal in the ${ }^{13} \mathrm{P}$ NMR of 2 at $\delta=-1.90(\mathrm{~s}, 1 \mathrm{P})$ were observed, confirming the $\alpha$ linkage between the disaccharide and phosphoric acid moieties. Deprotection of the benzyl group in $\mathbf{2}$ by hydrogenation over palladium catalyst gave $\mathbf{1 3}$ in $90 \%$ yield Scheme 2 . Coupling of $\mathbf{1 3}$ with UMP-morpholidate in the presence of $1 H$-tetrazole in DMFpyridine $(3: 1)^{16}$ followed by deprotection of the benzoyl groups and the methyl ester using $3 \mathrm{M} \mathrm{NaOH}$ afforded the target compound $\mathbf{1}^{17}$ in $45 \%$ yield over two steps after isolation and purification by reverse-phase column HPLC and gel-filtration column HPLC.

\section{Conclusions}

In summary, we have successfully accomplished the first chemical synthesis of $\mathrm{MeO}-3-\mathrm{GlcUA} \beta(1 \rightarrow 3)$ GlcNAc-UDP following a 


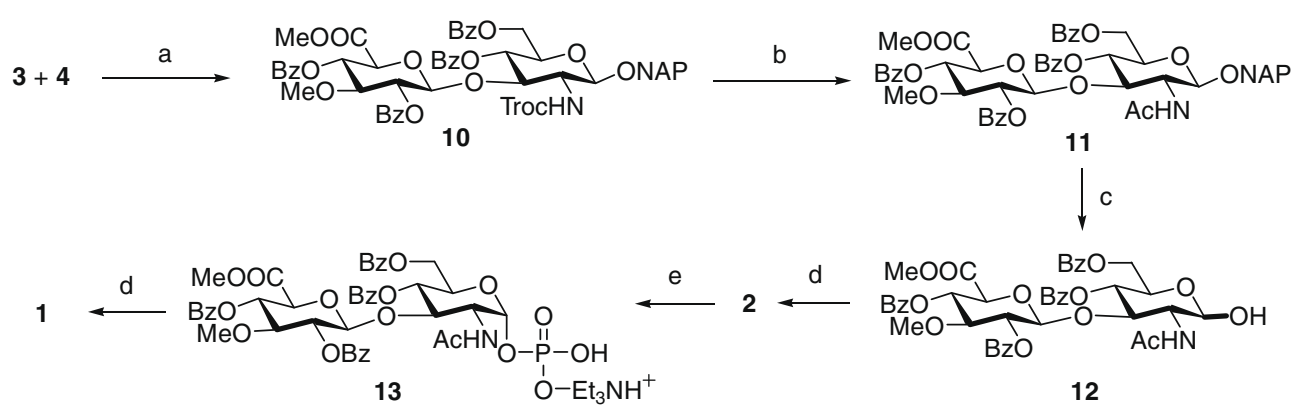

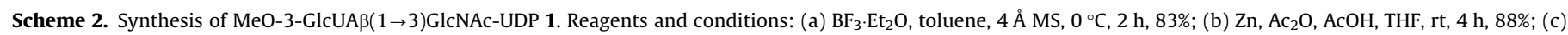

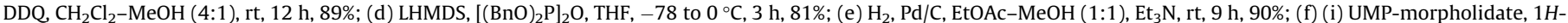
tetrazole, DMF-py (3:1), rt, 2 d; (ii) $3 \mathrm{M} \mathrm{NaOH}, \mathrm{MeOH}, \mathrm{rt}, 10 \mathrm{~h}$; (iii) RP column HPLC; gel-filtration column HPLC, $45 \%$ from 13.

very efficient glycosylation reaction which proceeded with complete stereoselectivity and high yield. We speculate that this compound would serve as a novel substrate to investigate the catalytic mechanism of HASs.

\section{Acknowledgments}

We acknowledge grant support from DOD (W81XWH-06-10013) and support, in part, by the NCI Cancer Center Support Grant to the Roswell Park Cancer Institute (P30-CA016056).

\section{References and notes}

1. (a) Laurent, T. C.; Fraser, J. R. E. FASEB J. 1992, 6, 2397-2404; (b) Knudson, C. B.; Knudson, W. FASEB J. 1993, 7, 1233-1241; (c) Zeng, C.; Toole, B. P.; Kinney, S. D.; Kuo, J.; Stamenkovic, I. Int. J. Cancer 1998, 77, 396-401; (d) DeAngelis, P. L. Cell. Mol. Life Sci. 1999, 56, 670; (e) Toole, B. P.; Wight, T. N.; Tammi, M. I. J. Biol. Chem. 2002, 277, 4593; (f) Toole, B. P. Nat. Rev. Cancer 2004, 4, 528; (g) Williams, K. J.; Halkes, K. M.; Kamerling, J. P.; DeAngelis, P. L. J. Biol. Chem. 2006 281, 5391.

2. Meyer, K.; Palmer, J. W. J. Biol. Chem. 1934, 107, 629-634.

3. (a) Prehm, P. Biochem. J. 2006, 398, 469; (b) Weigel, P. H.; DeAngelis, P. L. J. Biol. Chem. 2007, 282, 36777.

4. Termeer, C.; Benedix, F.; Sleeman, J.; Fieber, C.; Voith, U.; Ahrens, T.; Miyake, K.; Freudenberg, M.; Galanos, C.; Simon, J. C. J. Exp. Med. 2002, 195, 99-111.

5. (a) Slaghek, T. M.; Nakahara, Y.; Ogawa, T.; Kamerling, J. P.; Vliegenthart, J. F. G. Carbohydr. Res. 1994, 255, 61-85; (b) Sičinska, W.; Adams, B.; Lerner, L. Carbohydr. Res. 1993, 242, 29-51.

6. (a) Fowers, H. M.; Jeanloz, R. W. Biochemistry 1964, 3, 123-125; (b) Slaghek, T. M.; Hyppönen, T. K.; Kruiskamp, P. H.; Ogawa, T.; Kamerling, J. P.; Vliegenthart, J. F. G. Tetrahedron Lett. 1993, 34, 7939-7942; (c) Blatter, G.; Jacquinet, J.-C. Carbohydr. Res. 1996, 288, 109-120; (d) Garcia, B. A.; Poole, J. L.; Gin, D. Y. J. Am. Chem. Soc. 1997, 119, 7597-7598; (e) Yeung, B. K. S.; Hill, D. C.; Janicka, M.; Peuntilo, P. A. Org. Lett. 2000, 2, 1279-1282; (f) Crich, D.; Smith, M. J. Am. Chem. Soc. 2001, 123, 9015-9020; (g) Yeung, B. K. S.; Chong, P. Y. C.; Peuntilo, P. A Carbohydr. Res. 2002, 21, 779-865; (h) Codée, J. D. C.; Litjens, R. E. J. N.; Den Heeten, R.; Overkleeft, H. S.; Van Boom, J. H.; Van der Marel, G. A. Org. Lett. 2003, 5, 1519-1522; (i) Iyer, S. S.; Rele, S. M.; Baskaran, S.; Chaikof, E. L. Tetrahedron 2003, 59, 631-638; (j) Lu, X. A.; Chou, C. H.; Wang, C. C.; Hung, S. C. Synlett 2003, 9, 1364-1366; (k) Palmacci, E. R.; Seeberger, P. H. Tetrahedron 2004, 60, 7755-7766; (1) Codée, J. D. C.; Stubba, B.; Schiattarella, M.; Overkleeft H. S.; Van Boeckel, C. A. A.; Van Boom, J. H.; Van der Marel, G. A. J. Am. Chem. Soc 2005, 127, 3767-3773; (m) Huang, L.; Huang, X. Chem. Eur. J. 2007, 13, 529540; (n) Dinkelaar, J.; Codée, J. D. C.; Van den Bos, L. J.; Overkleeft, H. S.; Van der Marel, G. A. J. Org. Chem. 2007, 72, 5737-5742.

7. (a) Scott, M. E.; Viola, R. E. Carbohydr. Res. 1998, 313, 247-253; (b) Xia, J.; Xue, J.; Locke, R. D.; Chandrasekaran, E. V.; Srikrishnan, T.; Matta, K. L. J. Org. Chem. 2006, 71, 3696-3706; (c) Brown, J. R.; Yang, F.; Sinha, A.; Ramakrishnan, B.; Tor Y.; Qasba, P. K.; Esko, J. D. J. Biol. Chem. 2009, 284, 4952-4959.
8. Wei, G.; Kumar, V.; Xue, J.; Locke, R. D.; Matta, K. L. Tetrahedron Lett. 2009, 50, 5920-5922.

9. Brown, R. T.; Carter, N. E.; Mayalrap, S. P.; Scheinmann, F. Tetrahedron 2000, 56, 7591

10. Xue, J.; Khaja, S. D.; Locke, R. D.; Matta, K. L. Synlett 2004, 5, 861.

11. Hancock, G.; Galpin, I. J. Tetrahedron Lett. 1982, 23, 249.

12. (a) Yeung, B. K. S.; Hill, D. C.; Janicka, M.; Petillo, P. A. Org. Lett. 2000, 2, 1279; (b) Chen, L.; Kong, F. Carbohydr. Res. 2002, 337, 1373.

13. Kornilov, A. V.; Sukhova, E. V.; Nifantiev, N. E. Carbohydr. Res. 2001, 336, 309.

14. (a) Dullenkopf, W.; Castro-Palomino, J. C.; Manzoni, L.; Schmidt, R. R. Carbohydr. Res. 1996, 296, 135; (b) Schmidt, R. R.; Michel, J. Angew. Chem., Int. Ed. Engl. 1980, 19, 731.

15. (a) Schmidt, R. R. Angew. Chem., Int. Ed. Engl. 1986, 25, 212; (b) Allen, J. G.; Fraser-Reid, B. J. Am. Chem. Soc. 1999, 121, 468; (c) Rye, C. S.; Withers, S. G. J. Am. Chem. Soc. 2002, 124, 9756; (d) Rele, S. M.; Iyera, S. S.; Chaikof, E. L. Tetrahedron Lett. 2007, 48, 5055.

16. Takaku, H.; Ishida, H.-k.; Fujita, M.; Inazu, T.; Ishida, H.; Kiso, M. Synlett 2007, 818.

17. The selected physical data of key compounds are listed: Compound 3: $[\alpha]_{\mathrm{D}} 25$ $-12.5\left(\right.$ c 1.0, $\left.\mathrm{CHCl}_{3}\right) ;{ }^{1} \mathrm{H}$ NMR $\left(400 \mathrm{MHz}, \mathrm{CDCl}_{3}\right): \delta(\mathrm{ppm})=3.75(\mathrm{~s}, 3 \mathrm{H}), 3.82(\mathrm{~s}$, $3 \mathrm{H}), 4.22(\mathrm{t}, 1 \mathrm{H}, J=9.6 \mathrm{~Hz}), 4.41(\mathrm{~d}, 1 \mathrm{H}, J=9.6 \mathrm{~Hz}), 5.10(\mathrm{dd}, 1 \mathrm{H}, J=3.6,9.6 \mathrm{~Hz})$, $6.07(\mathrm{t}, 1 \mathrm{H}, J=10.2 \mathrm{~Hz}), 6.36(\mathrm{~d}, 1 \mathrm{H}, J=3.6 \mathrm{~Hz}), 7.35-8.01(\mathrm{~m}, 10 \mathrm{H}, \mathrm{Ph}), 8.74(\mathrm{~s}$, $1 \mathrm{H}) ;{ }^{13} \mathrm{C}$ NMR $\left(100 \mathrm{MHz}, \mathrm{CDCl}_{3}\right): \delta(\mathrm{ppm})=52.5,57.6,69.4,70.2,72.8,79.8$, 90.1, 92.3, 159.7, 167.5, 168.8, 169.1. ESI-HRMS: calcd for $\mathrm{C}_{24} \mathrm{H}_{22} \mathrm{Cl}_{3} \mathrm{NO}_{9} \mathrm{Na}$ $[\mathrm{M}+\mathrm{Na}]^{+}$596.0253, found: 596.0250. Compound 10: ${ }^{1} \mathrm{H}$ NMR (400 $\left.\mathrm{MHz}, \mathrm{CDCl}_{3}\right)$ : $\delta(\mathrm{ppm})=3.36(\mathrm{~s}, 3 \mathrm{H}), 3.40(\mathrm{~s}, 3 \mathrm{H}), 3.91-3.93(\mathrm{~m}, 1 \mathrm{H}), 4.32-4.34(\mathrm{~m}, 2 \mathrm{H}), 4.38$ $4.42(\mathrm{~m}, 2 \mathrm{H}), 4.48-4.52(\mathrm{~m}, 2 \mathrm{H}), 4.55-4.57(\mathrm{~m}, 1 \mathrm{H}), 4.82(\mathrm{~d}, 1 \mathrm{H}, J=8.2 \mathrm{~Hz}, \mathrm{H}-$ $\left.1^{\prime}\right), 5.02(\mathrm{~d}, 1 \mathrm{H}, J=8.0 \mathrm{~Hz}, \mathrm{H}-1), 5.06-5.13(\mathrm{~m}, 3 \mathrm{H}), 5.17(\mathrm{t}, 1 \mathrm{H}, J=9.6 \mathrm{~Hz}), 5.24$ $(\mathrm{t}, 1 \mathrm{H}, J=9.6 \mathrm{~Hz}), 5.34(\mathrm{bs}, 1 \mathrm{H}), 5.50(\mathrm{t}, 1 \mathrm{H}, J=9.6 \mathrm{~Hz}), 7.20-8.20(\mathrm{~m}, 27 \mathrm{H}) ;{ }^{13} \mathrm{C}$ $\operatorname{NMR}\left(100 \mathrm{MHz}, \mathrm{CDCl}_{3}\right): \delta(\mathrm{ppm})=53.5,57.2,58.7,63.5,70.5,70.9,71.3,71.8$ 72.1 (2C), 72.7, 74.6, 80.8, 95.6, 99.8, 100.3, 153.2, 165.2, 166.8, 167.1, 169.0, 169.7; ESI-HRMS: calcd for $\mathrm{C}_{56} \mathrm{H}_{50} \mathrm{Cl}_{3} \mathrm{NO}_{17} \mathrm{Na}[\mathrm{M}+\mathrm{Na}]^{+} 1136.2037$, found: 1136.2035. Compound 2: ${ }^{1} \mathrm{H}$ NMR $\left(400 \mathrm{MHz}, \mathrm{CDCl}_{3}\right): \delta(\mathrm{ppm})=1.83(\mathrm{~s}, 3 \mathrm{H})$, $3.31(\mathrm{~s}, 3 \mathrm{H}), 3.51(\mathrm{~s}, 3 \mathrm{H}), 3.82(\mathrm{~d}, J=10.0 \mathrm{~Hz}, 1 \mathrm{H}), 4.17(\mathrm{t}, J=9.8 \mathrm{~Hz}, 1 \mathrm{H}), 4.21-$ $4.26(\mathrm{~m}, 2 \mathrm{H}), 4.39-4.49(\mathrm{~m}, 2 \mathrm{H}), 4.67-4.74(\mathrm{~m}, 2 \mathrm{H}), 4.97-5.10(\mathrm{~m}, 6 \mathrm{H}), 5.40(\mathrm{t}$, $J=8.8 \mathrm{~Hz}, 1 \mathrm{H}), 5.68\left(\mathrm{dd}, J_{12}=3.4 \mathrm{~Hz}, J_{1 \mathrm{p}}=6.4 \mathrm{~Hz}, 1 \mathrm{H}, \mathrm{H}-1\right), 5.76(\mathrm{~d}, J=9.2 \mathrm{~Hz}$, NHAc), 7.26-8.20 (m, 30H). ${ }^{13} \mathrm{C} \mathrm{NMR}\left(100 \mathrm{MHz}, \mathrm{CDCl}_{3}\right): \delta(\mathrm{ppm})=20.1,51.7$, 52.3, 57.8 (JC-2,P $=8.1 \mathrm{~Hz}, \mathrm{C}-2), 62.1,68.2,68.7,69.4\left(\mathrm{~J}_{\mathrm{CH} 2 \mathrm{Ph}, \mathrm{P}}=4.9 \mathrm{~Hz}, \mathrm{POCH}_{2} \mathrm{Ph}\right)$, $69.7\left(J_{\mathrm{CH} 2 \mathrm{Ph}, \mathrm{P}}=4.9 \mathrm{~Hz}, \mathrm{POCH}_{2} \mathrm{Ph}\right), 71.6,72.4,73.8,74.2,79.8,96.4\left(J_{\mathrm{C}}\right.$ $\left.{ }_{1 \mathrm{P}}=6.9 \mathrm{~Hz}, \mathrm{C}-1\right), 99.4\left(\mathrm{C}-1^{\prime}\right), 164.4,165.5,166.4,168.6,168.9,169.4 .{ }^{31} \mathrm{P}$ NMR $\left(202 \mathrm{MHz}, \mathrm{CDCl}_{3}\right): \delta(\mathrm{ppm})=-1.90$ (s, 1P). ESI-HRMS: calcd for $\mathrm{C}_{58} \mathrm{H}_{56} \mathrm{NO}_{19} \mathrm{PNa}[\mathrm{M}+\mathrm{Na}]^{+} 1124.3077$, found: 1124.3080 . Compound 1: ${ }^{1} \mathrm{H}$ NMR $\left(400 \mathrm{MHz}, \mathrm{D}_{2} \mathrm{O}\right): \delta(\mathrm{ppm})=1.93(\mathrm{~s}, 3 \mathrm{H}), 3.53(\mathrm{~s}, 3 \mathrm{H}), 3.56(\mathrm{~m}, 1 \mathrm{H}), 3.60(\mathrm{t}$, $J=9.6 \mathrm{~Hz}, 1 \mathrm{H}), 3.63-3.88(\mathrm{~m}, 6 \mathrm{H}), 3.93(\mathrm{~d}, J=9.2 \mathrm{~Hz}, 1 \mathrm{H}), 4.07$ (ddd $\left.J_{2,1}=3.4 \mathrm{~Hz}, J_{2,3}=10.2 \mathrm{~Hz}, J_{2, \mathrm{P}}=3.2 \mathrm{~Hz}, 1 \mathrm{H}, \mathrm{H}-2\right), 4.10-4.13(\mathrm{~m}, 2 \mathrm{H}), 4.15-4.18$ $(\mathrm{m}, 1 \mathrm{H}), 4.20-4.24(\mathrm{~m}, 2 \mathrm{H}), 4.31(\mathrm{t}, J=9.6 \mathrm{~Hz}, 1 \mathrm{H}), 4.43\left(\mathrm{~d}, J_{1^{\prime}, 2^{\prime}}=8.6 \mathrm{~Hz}, 1 \mathrm{H}, \mathrm{H}-\right.$ $\left.1^{\prime}\right), 5.40\left(\mathrm{dd}, J_{1,2}=3.2 \mathrm{~Hz}, J_{1, \mathrm{p}}=7.8 \mathrm{~Hz}, 1 \mathrm{H}, \mathrm{H}-1\right), 5.79(\mathrm{~d}, 1 \mathrm{H}), 5.83(\mathrm{~d}, 1 \mathrm{H}), 7.83$ $(\mathrm{d}, 1 \mathrm{H}) .{ }^{13} \mathrm{C}$ NMR $\left(100 \mathrm{MHz}, \mathrm{D}_{2} \mathrm{O}\right): \delta(\mathrm{ppm})=23.4,54.5,57.6\left(J_{\mathrm{C}-2, \mathrm{P}}=8.6 \mathrm{~Hz}, \mathrm{C}-\right.$ 2), 62.8, 66.0 (d, $J=3.8 \mathrm{~Hz}$ ), 70.0, 71.1, 71.6, 72.5, 74.2, 74.8, 75.5, 77.2, 81.2, $84.8(\mathrm{~d}, J=9.8 \mathrm{~Hz}), 89.5,96.5\left(J_{\mathrm{C}-1 . \mathrm{P}}=6.0 \mathrm{~Hz}, \mathrm{C}-1\right), 101.3,104.1,141.8,153.4$, 167.4, 175.5, 176.1. ${ }^{31} \mathrm{P}$ NMR $\left(202 \mathrm{MHz}, \mathrm{D}_{2} \mathrm{O}\right): \delta(\mathrm{ppm})=-11.4(\mathrm{~d}, J=22.4 \mathrm{~Hz})$, -13.1 (d, $J=22.6 \mathrm{~Hz}$ ). ESI-HRMS: calcd for $\mathrm{C}_{24} \mathrm{H}_{36} \mathrm{~N}_{3} \mathrm{O}_{23} \mathrm{P}_{2}[\mathrm{M}-\mathrm{H}]^{-}$796.1210, found: 796.1214 